\title{
DESIGN AND IMPLEMENTATION OF A SOFTWARE COMPONENT FOR GEOSPATIAL DATA VISUALIZATION IN EXCEL
}

\author{
Marian DÂRDAL $\breve{A}$ \\ Bucharest University of Economic Studies \\ dardala@ase.ro \\ Felix Titus FURTUNĂ \\ Bucharest University of Economic Studies \\ titus@ase.ro \\ Cristian IONIȚĂ \\ Bucharest University of Economic Studies \\ cristian.ionita@ie.ase.ro
}

\begin{abstract}
This paper proposes an architecture for a software component that allow us to display data from an xlsx document in a geospatial context. In order to spatial data manipulation and visualization, it was used specialized libraries as Geopandas and Matplotlib in a user defined Python script. The software component is plugged to Excel application as an Add-in, in this case it can be used in any document is necessary. To emphasize the utility of this component in the end of this paper is presented a case study for displaying the share of urban population for each Romanian county on a map.
\end{abstract}

Keywords: Spatial Visualization, GIS, Excel, Software Interoperability and Integration. JEL classification: Y100, C880

DOI: $10.12948 / \mathrm{ie} 2019.04 .22$

\section{Introduction}

It is well known that many organizations have a lot of data stored in $x l s x$ documents and process them using Excel computation techniques. A large number of users even non-IT specialists uses these techniques. Besides this, in Excel, data can be presented in various graphical forms using the chart control but, in many cases, data reflects spatial phenomena and it is very useful to display them in geospatial context using maps [10]. There are map controls that use geographical data from online platforms such as Bing Maps or ArcGIS online and can be incorporated in Excel spreadsheets. The control we designed and implemented combines spatial data created with GIS tools $[7,8]$ and stored in an independent format (Shape File $s h p$ ) with the non-spatial data stored in spreadsheets. This manner of working with spatial data in Excel offers at least two advantages: the users can use more accurate spatial data and doesn't require an Internet connection.

\section{Development of software applications based on MSOffice}

There are many ways to develop applications based on Office: create an independent application that uses Office applications in background, build a software component plugged to an Office application as Add-in as well as use code behind document technique to attach code to process data from a specific document. In order to communicate with Office application and using specific entities from Office documents we used Visual Studio Tools for Office (VSTO) framework [1].

The existing controls are available in Excel as Add-in components: 
- Microsoft Power Map for Excel [11] is built as an extension that has to be enabled before use it and allow us to work with maps in 3D form I mean as globe. In the Insert ribbon is 3DMap button by clicking the user can start to work with the component. The map visualization window is shown in the figure 1. Excel sheets have to contain spatial references as coordinates (latitude / longitude), countries, cities, regions; postal addresses and so on in order to be represented on Bing Maps and other parameters in relation with spatial data can be displayed in various forms on the map.

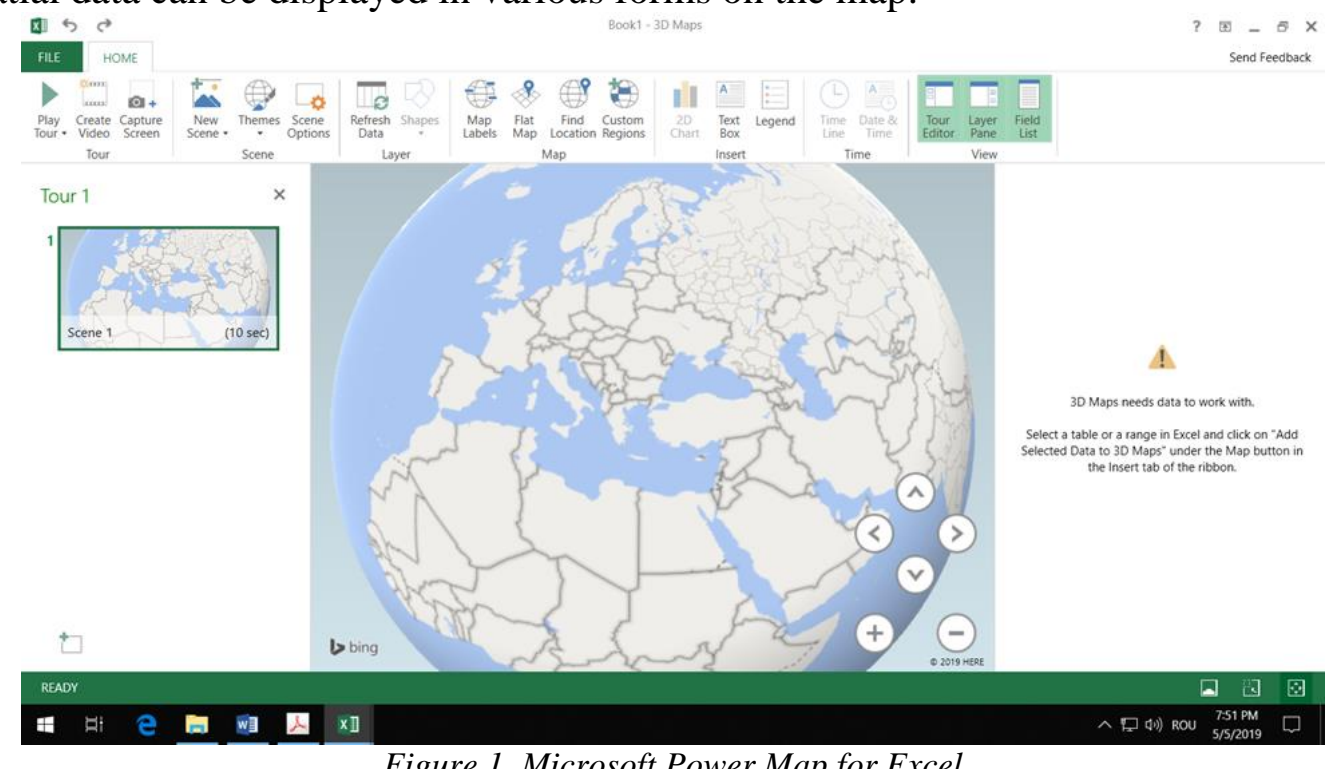

Figure 1. Microsoft Power Map for Excel

- ArcGIS Maps for Office [3] is developed by ESRI and provides support for Excel and PowerPoint applications. This component works with spatial data form ArcGIS Online and the spreadsheets have to contain spatial references and other data for displaying on map, organized in tables. For instance, in the figure 2 is presented a map that shown the total population on the Romanian counties using a graduated colors symbology. As spatial references we used the counties names. The user can use his own content as Web GIS application [9] from ArcGIS online to be viewed in the control and he can interact with it.

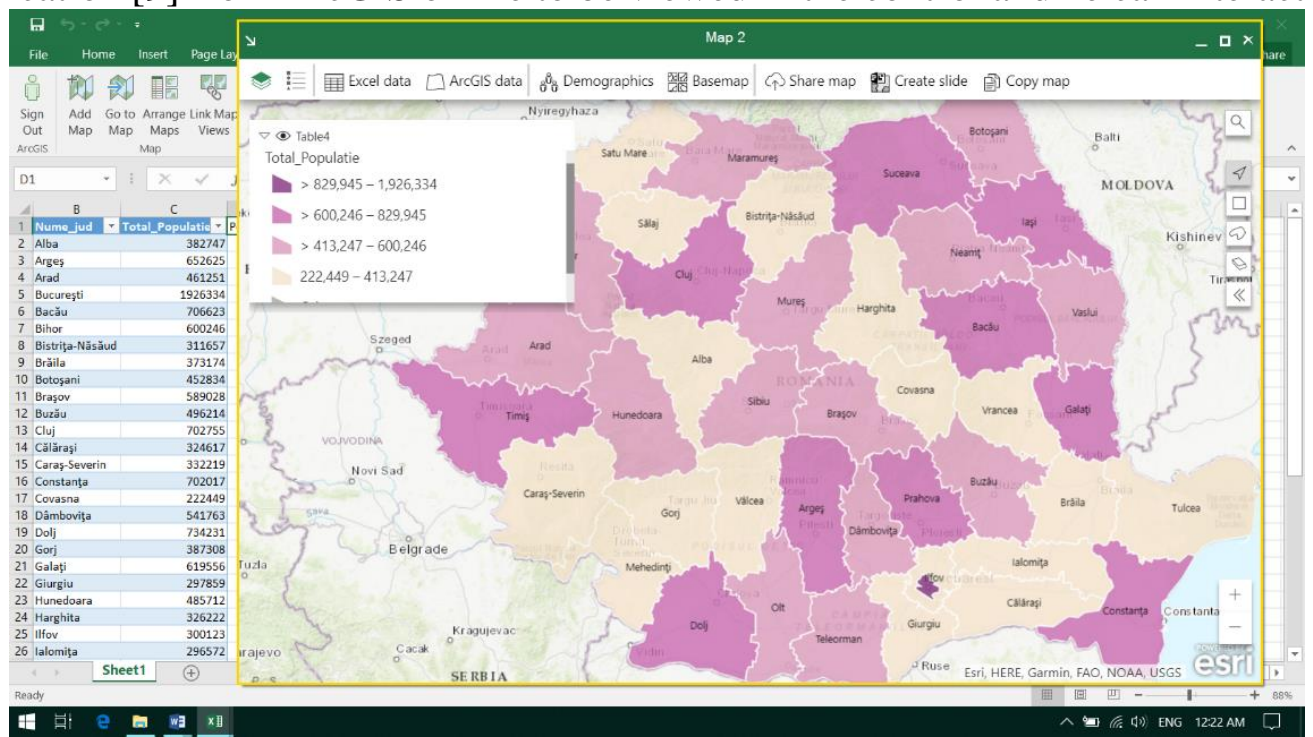

Figure 2. ArcGIS Maps for Office

The Create slide button generates a new slide in PowerPoint with the image of the map. 
www.conferenceie.ase.ro

\section{Design an Excel plugin for geospatial visualization}

The control we designed and implemented combines and integrates [2] different software technologies. The Add-in for Excel was developed in C\# using VSTO framework [1]. Spatial data manipulation and visualization was implemented in Python with the help of specialized libraries [5,6]. A Dynamic Link Library (DLL) that contains exportable C functions makes the link between the Add-in and the Python script using the Python/C Application Programming Interface (API) [12]. By using PInvoke (Platform Invocation Services) mechanism [4], the unmanaged code from the DLL was called from the managed code Add-in component. Figure 3 presents the architecture of the application.

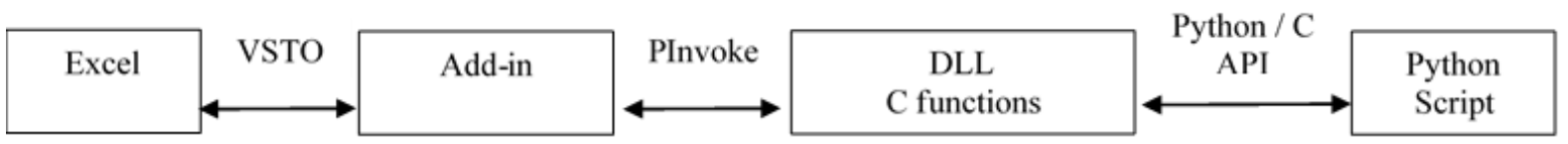

Figure 3. The Architecture of the software module

In Excel the interface of this Add-in resides in the Geo_vizualizare ribbon, which contains the GIS group and the Afisare_Harta button as in figure 4.

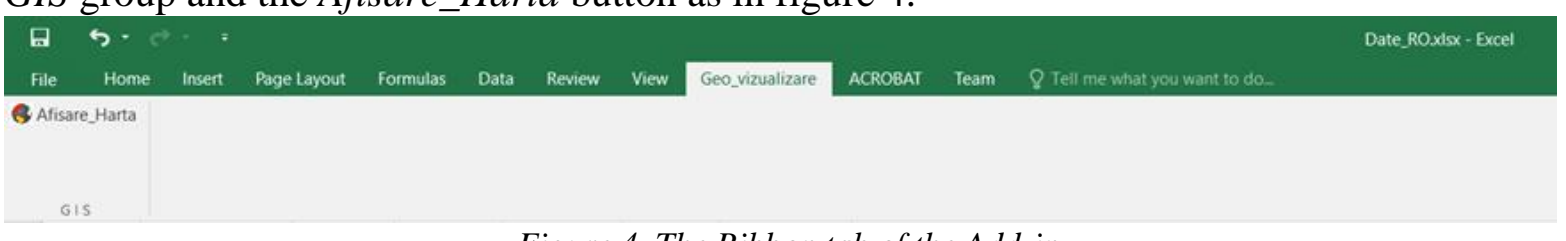

Figure 4. The Ribbon tab of the Add-in

Before displaying the map in the sheet, the user has to select data and configure the visualization. Figure 5 shows the form that allows to the user to set up all visualization parameters necessary for obtaining the desired result. Data selection is for non-spatial data from sheet and for spatial data from external source. It is important to establish an attribute from the shp file and a range from sheet to realize the matching between spatial data and non-spatial ones. Taking into consideration the map display configuration, the user can mark the cell in sheet that locate top-left corner of the image, choose whether to display axes or not, select labels on spatial data and choose the symbology (graduated colors for a continuous value domain or discrete colors for discrete values).

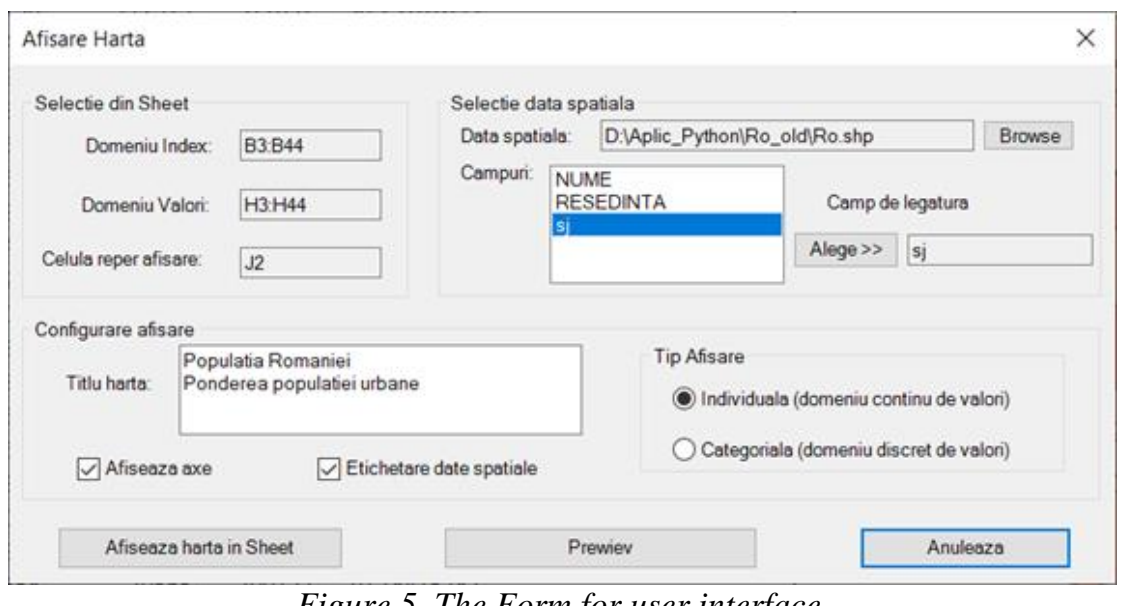

Figure 5. The Form for user interface

The functionality of the software component is the following: data collected from the sheet is prepared and sent to the Python script to generate the map as image in png format, then it is displayed in the sheet. The main Python function that generates the image is viz_harta:

import pandas as pd

import geopandas as gp

import matplotlib.pyplot as plt 


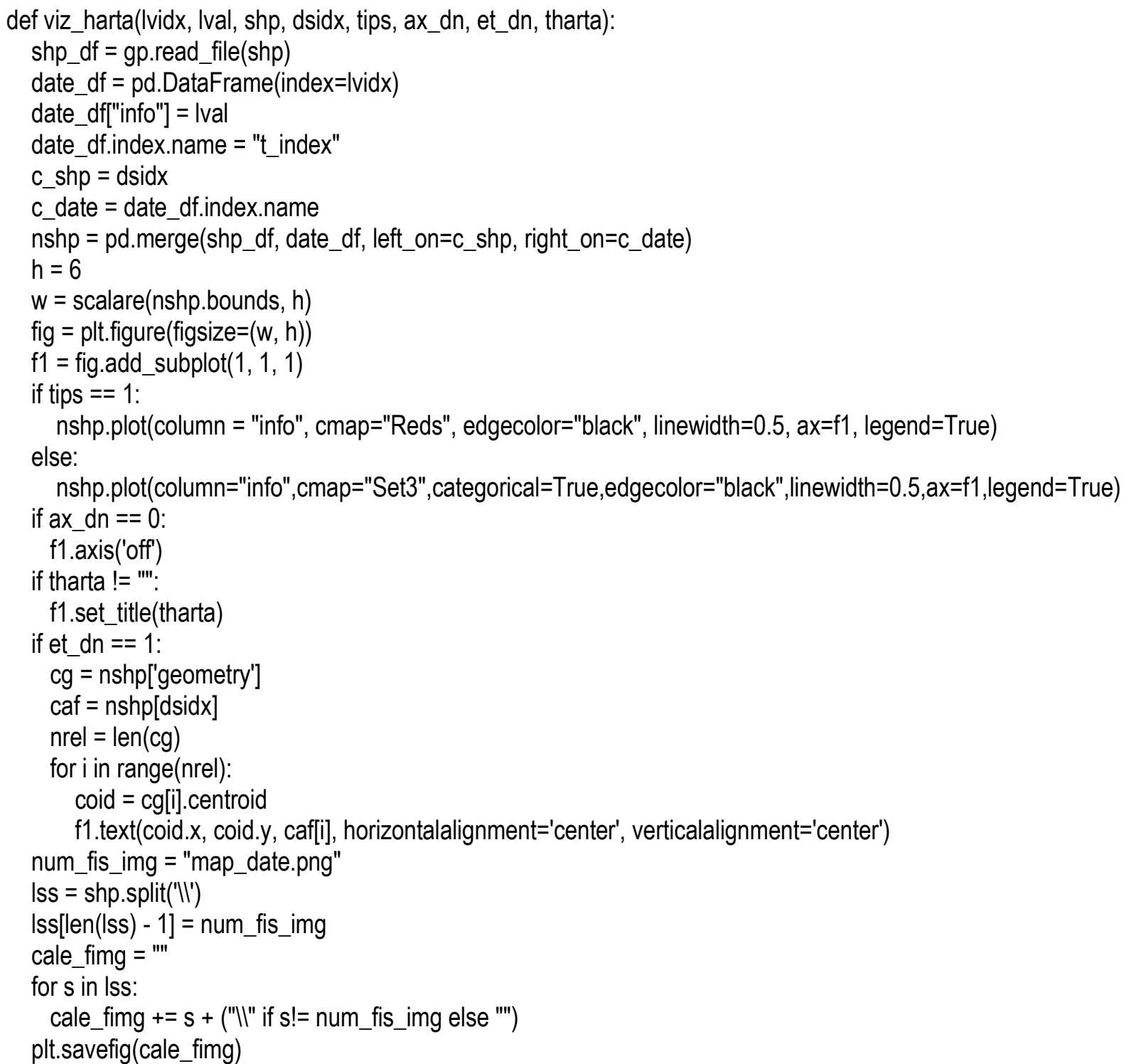

The code sequence that displays the image of the map in the sheet is:

Excel. Worksheet wsh = f1eapl.ActiveSheet;

Excel.Range rng_ss_imag = wsh.get_Range(tb_img.Text);

wsh.Shapes.AddPicture(cale_img, Microsoft.Office.Core.MsoTriState.msoFalse,

Microsoft.Office.Core.MsoTriState.msoCTrue, rng_ss_imag.Left, rng_ss_imag.Top, -1, -1);

where fleapl is the Excel application object and tb_img.Text indicates the cell that marks the position of the image in the sheet.

\section{Case study: Romanian population at county level by origin}

Per county data is stored in an Excel spreadsheet (figure 6) using the following structure:

- county code;

- population living in cities (over 100000 inhabitants);

- population living in towns;

- population living in rural areas.

The goal of the case study is to represent on a map the share of the urban population in the total population at the level of each county using a gradual color symbology. 
www.conferenceie.ase.ro

Using Excel for data storage and manipulation has the advantage compared to traditional GIS tools of allowing non-specialist users to access, transform and present the information using the instruments they're already familiar with.

In this context, the urban population (Pop urban) was calculated at the level of each county, as the sum of population in cities and the population in towns and the result was stored in a distinct column. The total population (Total Pop) of the county was calculated by adding the urban population to the rural population. Finally, the share of urban population (Pondere) in the total population for each county was calculated as:

$$
\text { Pondere }_{i}=\frac{\text { Pop urban }_{i}}{\text { Total Pop }_{i}}, \quad \text { where } i=\overline{1,42}
$$

(Romania has 41 counties and a city with special status - Bucharest.

After the calculation of the indicators, the spatial mapping of the weights was carried out using the presented Excel Add-In and a graduated color symbology (highly urbanized areas are using darker red tones and more rural areas are using lighter reds). The visualization reflects two aspects. The first, which are the most urbanized counties, could be graphically expressed through a histogram. On the other hand, the localization on the territory of Romania and the geographical relationship between them is impossible to convey using standard Excel charts. The resulted map has been integrated into the Excel spreadsheet as an image (figure 6). There it can be resized, moved, deleted, or stored with the document in the $x l s x$ file.

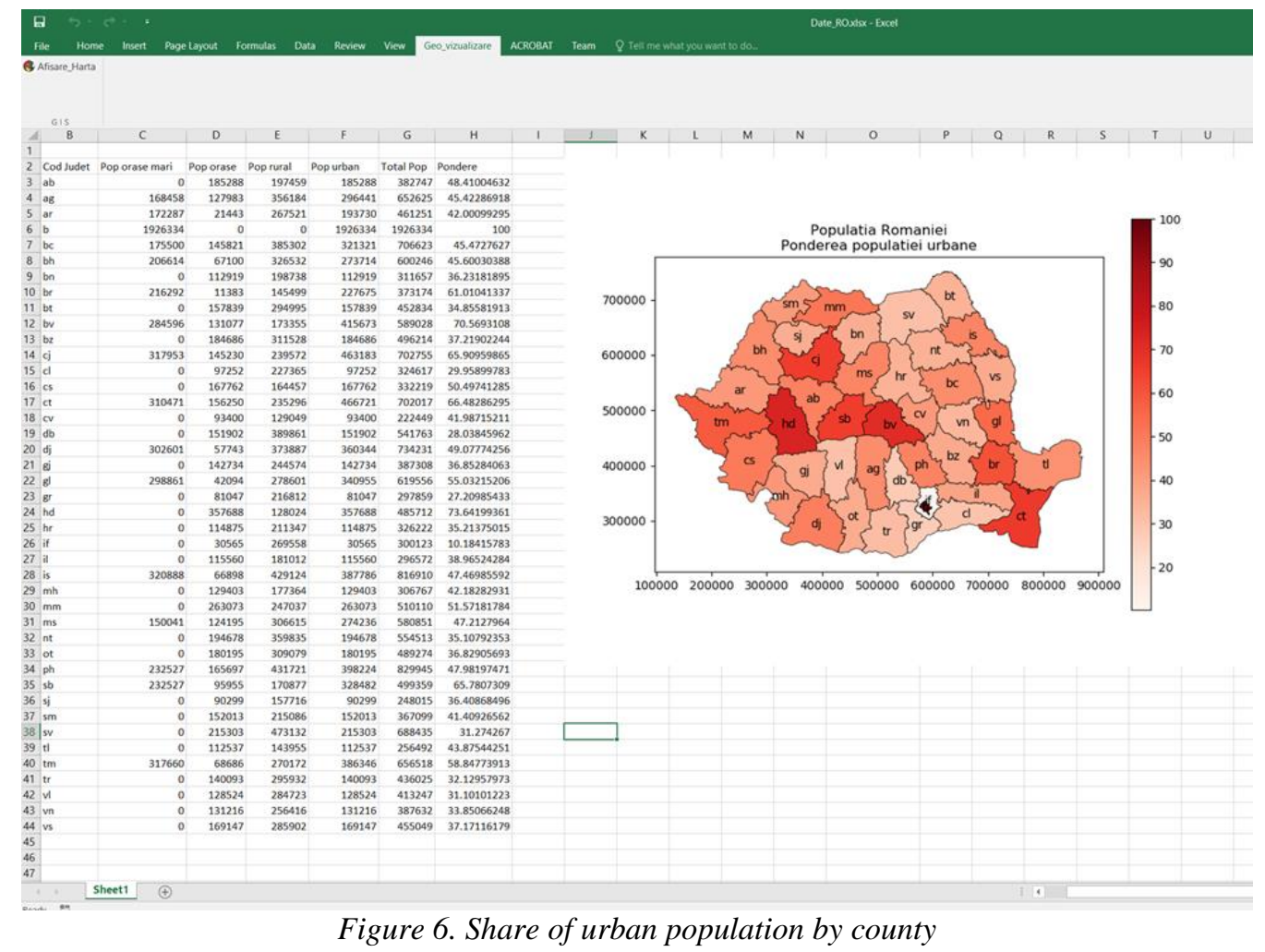

The visualization allows us to draw the following conclusions:

- The city of Bucharest, being the largest city in the country, is an extreme case because it has a $100 \%$ urban population.

- A large percentage of the urban population, about $73 \%$, is found in Hunedoara County, although the county has no big cities, but it has many cities around the small mining towns such as Lupeni, Petrila, Vulcan, Aninoasa, Calan, Uricani, Petrosani etc. 
www.conferenceie.ase.ro

- Most of the counties with a share of the urban population (over 60\%) are concentrated in the Ardeal province (Brasov, Sibiu, Cluj, Timis), while the rest of the country contains mostly rural counties with the notable exceptions of Constanta and Braila counties.

\section{Conclusions}

Software development technologies allow us to extend the functionality of the software packages build using extensible frameworks. In this way the applications can be extended to satisfy a larger range of use cases make users more productive. The component presented in this paper can be improved in different ways to ensure a complete functionality concerning map creation and visualization.

\section{References}

[1] E. Carter and E. Lippert,Visual Studio Tools for Office 2007, Addison-Wesley, USA, 2009;

[2] M. Dârdală, T.F. Furtună and A. Reveiu, INTEGRATING R SCRIPTS IN GIS, The 15th International Conference on Informatics in Economy (IE 2016), Education, Research \& Business Technologies, Cluj-Napoca, ROMANIA, 2016;

[3] M. Flanagan, ArcGIS Maps for Office: Your Maps in Excel and PowerPoint, http://proceedings.esri.com/library/userconf/fed17/papers/fed_08.pdf;

[4] S. Goldshtein, D. Zurbalev and I. Flatow, Unsafe Code and Interoperability, In: Pro .NET Performance, Apress, Berkeley, CA, 2012, https://doi.org/10.1007/978-1-4302-4459-2_8

[5] J. Lawhead, Learning Geospatial Analysis with Python - Second Edition, O'Reilly - Packt Publishing, 2015;

[6] W. McKinney, Python for Data Analysis, 2nd Edition, O’Reilly, 2017;

[7] B. Mihai, I. Şandric and Z. Chiţu, Some contributions to the drawing of the general geomorphic map using GIS tools. An application to Timis Mountains, Revista de Geomorfologie, vol 10, no 1, 2008;

[8] A. Omran, S. Dietrich, A. Abouelmagd and M. Michael, New ArcGIS tools developed for stream network extraction and basin delineations using Python and java script, COMPUTERS \& GEOSCIENCES , Vol. 94, 2016;

[9] A. Reveiu, A WEB GIS SOLUTION FOR IDENTIFYING AND ANALYSIS CLUSTER TYPE AGGLOMERATIONS FROM TOURISM INDUSTRY, The 16th International Conference on Informatics in Economy (IE 2017), Education, Research \& Business Technologies, Bucharest, Romania, 2017;

[10] M. Wohlstadter, L. Shoaib, J. Posey, J. Welsh and J. Fishman, A Python toolkit for visualizing greenhouse gas emissions at sub-county scales, ENVIRONMENTAL MODELLING \& SOFTWARE, Vol. 83, 2016;

[11] Microsoft Power Map Preview for Excel, http://egis3.lacounty.gov/eGIS/wpcontent/uploads/2015/03/Started-with-Power-Map-Preview.pdf;

[12] Python/C API Reference Manual, https://docs.python.org/3/c-api/. 